\title{
Learning from learning algorithms: Application to attosecond dynamics of high-harmonic generation
}

\author{
Randy A. Bartels \\ Department of Electrical and Computer Engineering, Colorado State University, Ft. Collins, Colorado, USA \\ Margaret M. Murnane* and Henry C. Kapteyn \\ JILA, University of Colorado, Boulder, Colorado 80309-0440, USA \\ Ivan Christov \\ Department of Physics, Sofia University, Sofia, Bulgaria \\ Herschel Rabitz \\ Department of Chemistry, Princeton University, Princeton, New Jersey, USA
}

(Received 15 January 2004; published 13 October 2004)

\begin{abstract}
Using experiment and modeling, we show that the data set generated when a learning algorithm is used to optimize a quantum system can help to uncover the physics behind the process being optimized. In particular, by optimizing the process of high-harmonic generation using shaped light pulses, we generate a large data set and analyze its statistical behavior. This behavior is then compared with theoretical predictions, verifying our understanding of the attosecond dynamics of high-harmonic generation and uncovering an anomalous region of parameter space.
\end{abstract}

DOI: 10.1103/PhysRevA.70.043404

PACS number(s): 32.80.Qk, 42.62.-b, 89.20.-a

Experiments that study the dynamics of quantum systems, such as optical studies of atomic and molecular dynamics, often employ a "pump-probe" configuration where a pump pulse perturbs a system, and a probe pulse at varying time delay probes its evolution [1]. However, this technique corresponds to a simplified case of the more-general "stimuliresponse" experiment, where by observing the dynamical response of a system to varying stimulus, one can compare experiment with hypothesis. Often, in the case where the physics of a system is relatively simple, a pump-probe experiment can provide the most-readily interpretable data. However, pump-probe experiments can be difficult to interpret in the case of a complex quantum system, where the full dynamics are not already understood. Therefore devising new approaches to uncover and understand the dynamics of quantum systems is very important, in particular in the case of complex chemical and biological systems. Another area that requires a more sophisticated approach is the emerging field of "attosecond science" where subfemtosecond electron dynamics in atoms and molecules are observed. In this area, experimental constraints limit the applicability of a straightforward "pump-probe" experiment, and instead experiments infer various light source properties and electronic dynamics, using various comparisons of experimental observations with theoretical models [2-5].

The more general case of a stimulus-response experiment was discussed by Rabitz [6] for the case of optically probed quantum (i.e., atomic, molecular, or electronic) systems. He suggested that the use of "learning algorithms" could both

*FAX: (303) 492-5235.

Electronic address: murnane@jila.colorado.edu accomplish coherent control over a quantum system to obtain a desirable outcome [7-12], as well as provide information about the quantum system itself. In past work, we demonstrated the power of learning algorithms to selectively optimize the generation of coherent extreme-ultraviolet light using high-harmonic generation (HHG) [2]. By adjusting the phase of the laser field guided by a learning algorithm, we manipulated and optimized the quantum interferences that occur during the HHG process to achieve selective optimization of a single harmonic order. A comparison of theory with experiment was used to identify the mechanism behind the optimization: an optimally shaped light pulse allowed the phase of the radiating electron wave function to be adjusted on 10-20 attosecond time scales to selectively optimize a single harmonic order $[13,14]$. Both the optimization result itself that allowed selective maximization of a particular harmonic, as well as the mechanism behind the optimization, were not anticipated in advance of the experiment. Therefore new approaches that allow further validation of theoretical models used in optimal control experiments are highly desirable.

In this work we show that by analyzing the statistical behavior of the trial solutions generated during the process of optimizing high-harmonic generation, we can independently corroborate the mechanism behind the optimization, as well as uncover interesting behavior for a quantum system. The learning algorithm finds and probes an interesting, unexpected, and previously unobserved region of parameter space where a single harmonic order is selectively optimized, and where the chirps of adjacent harmonics have opposite sign. All previous work demonstrated adjacent harmonic orders to have similar chirps $[15,16]$. This anomalous chirp behavior found by the learning algorithm leads to an observed anticor- 
relation in intensity between adjacent harmonic orders that can be used to validate theory through a statistical analysis of the data set. More generally, our analysis [17] and recent parallel work on other systems $[18,19]$ show that the entire data set acquired during an optimization process is useful for understanding the physical system under investigation. In a learning algorithm experiment, the algorithm iteratively performs thousands of experiments and observes the results of each. Thus in the process of training the experimental apparatus to optimize a quantum process we acquire a great deal of information about the dynamics of the quantum system. An analysis of the optimization pathway itself thus serves to probe the dynamics and can be used to independently compare with theoretical predictions. Understanding what can be learned from optimal control data sets is particularly useful in the case of nonperturbative, high-field phenomena that are not well understood.

HHG is a coherent, nonlinear, optical process. In HHG, a large number of visible-energy photons from an intense laser pulse are combined into a single high-energy photon. The semiclassical theory of HHG considers an atom irradiated by an intense, ultrashort light pulse. At intensities approaching $10^{14} \mathrm{~W} \mathrm{~cm}^{-2}$, the optical field is sufficiently strong that the Coulomb barrier binding the outermost electron to the atom is suppressed. Electrons can then tunnel through the barrier, leading to field ionization of the atom. This process occurs twice per optical cycle as the optical field strength peaks. Once ionized, the electrons oscillate in the light field and some fraction of the ionized electrons recollide with the parent ion and recombine with it, releasing their kinetic energy as a high-energy photon. The shape of the driving laser pulse determines the trajectory of the electron as it oscillates in the laser field, and hence the quantum phase accumulated during its trajectory, as well as the interferences that occur between different adjacent electron trajectories. This quantum phase in turn determines the harmonic phase and ultimately the spectrum of the generated high-harmonic radiation.

In our experiment, mJ-energy pulses from a $1-\mathrm{kHz}$ repetition-rate laser system [20] were focused into a 175 - $\mu$ m-diameter argon-filled hollow waveguide. This creates a phase-matched comb of harmonics containing the 23rd-31st orders [21]. The waveguide geometry creates an extended region of high laser intensity and long coherence length to efficiently generate the high-harmonic radiation, which is observed using a spectrometer- $\mathrm{X}$-ray charge-coupled device camera system. A deformable mirror pulse shaper [22] and an evolutionary strategy algorithm were then used to selectively optimize the 27 th harmonic, while simultaneously suppressing the 25th and 29th orders. The optimum pulse shape is found after about 100 iterations of the learning algorithm, each of which consists of about 100 trials.

Although the learning process is fundamentally "trial and error," this does not mean that the pathway the learning algorithm takes toward optimization is a random one. In particular, there are very significant changes in the statistical behavior of the "optimum" solution as the optimization proceeds, reflecting the guidance provided by the learning algorithm. Figure 1(a) shows the peak intensity of the 25th, 27 th, and 29th harmonic orders for the optimal pulse shape from each iteration of the learning algorithm, where the learning

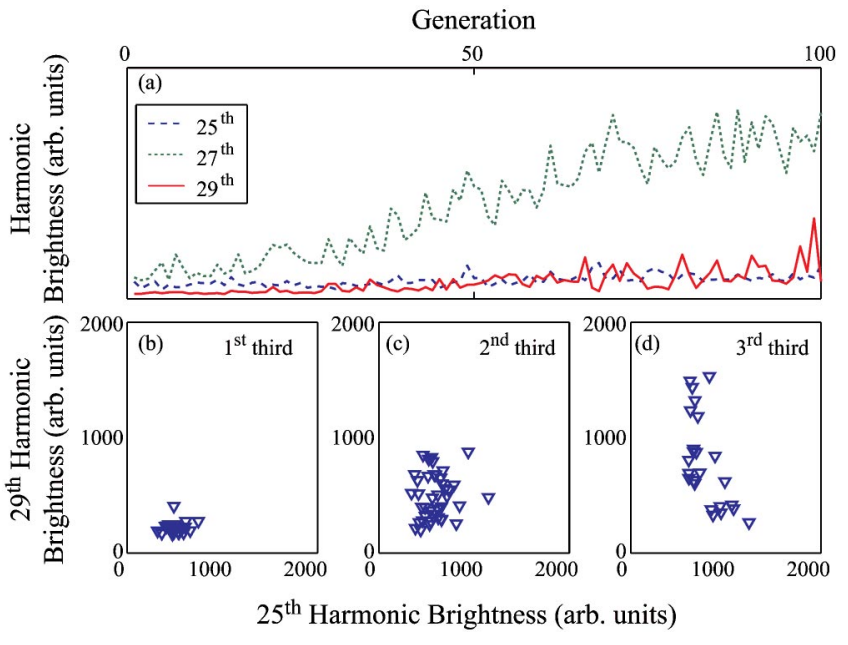

FIG. 1. (a) Peak brightness of the 25th, 27th, and 29th, harmonic orders from an experiment to selectively optimize the 27 th order. A correlation map of the 25th and 29th harmonic orders for the first (b), second (c), and third (d) fractions of the learning algorithm experiment.

algorithm fitness function, $f=1-\left(b_{25}+b_{29}\right) / 2 b_{27}$, is constructed to selectively optimize the 27 th harmonic, increasing the intensity contrast between the 27th and the neighboring harmonic orders. Here $b_{i}$ is the brightness of the $i$ th harmonic. The correlation between the intensities of the 25th and 29th harmonic orders is plotted in Figs. 1(b)-1(d) for the first, second, and third fractions of the data set. Initially, [Fig. 1(b)] the pulse shapes that correspond to the trial population are random. However, after a few iterations there exists a population of shapes that largely do generate a highharmonic spectrum. Furthermore, the initial pulse shapes generally do not selectively enhance a particular harmonic order in comparison with the relative harmonic magnitudes obtained from a transform-limited pulse. In this early stage, the intensity of all harmonic orders fluctuates together; i.e., the intensities are correllated. However, by the end of the optimization process [Fig. 1(d)], the intensity of the 27th harmonic has been enhanced, and the intensities of the 25th and 29th harmonics are strongly anticorrelated.

We can quantify this relationship by using Spearman's rho- a correlation function that enumerates correlated trends in pairwise data [23]. If both monotonically increase, the correlation is 1 , while if one increases and the other decreases, the value is -1 (anticorrelated case). The value of Spearmann's rho for the first four iterations of the learning algorithm is +0.80 and +0.54 for the first six generations, indicating the neighboring harmonic orders exhibit a strong positive correlation. This positive correlation between the neighboring harmonic orders indicates that the brightness of both of the neighboring harmonic orders either increase or decrease in response to pulse shape perturbations. However, as the algorithm progresses, it begins to find pulse shapes that exhibit the desired harmonic-order selectivity, and this is also accompanied by changes in the statistical correlations of the HHG spectra, in which the 25th and 29th order harmonics become anticorrelated. This indicates that the learning algorithm has found a different region of parameter space for 


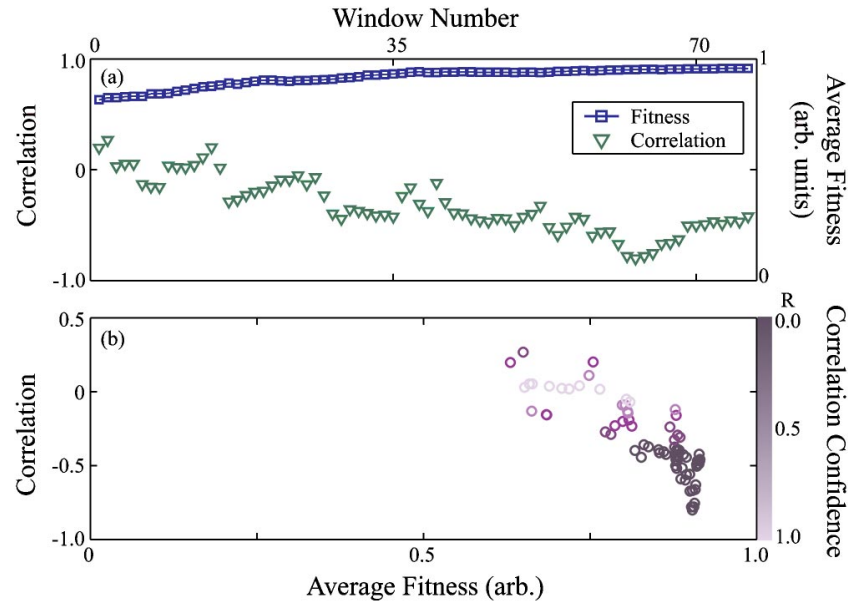

FIG. 2. (a) Correlation of the 25th and 29th experimental harmonic brightness as a moving window (15 generations wide) is scanned along algorithm generations (triangles) and the corresponding average fitness value (points). (b) Correlations plotted vs the average fitness value for the data in (a), where the color is graded from dark to light for high to low confidence in the correlation result.

driving HHG. We can observe this transition by calculating Spearmann's rho for a moving window across the data set as shown in Fig. 2(a) (triangles); note that the window width of 15 was chosen to maximize the average confidence of the statistical analysis (most of the statistics exhibited a confidence of $>90 \%$ in the calculated correlation value) across the data set. After only $\sim$ ten generations, the data become anticorrelated. This anticorrelation is accompanied by an increase in the fitness [Fig. 2(a), points], indicating that the harmonic selectivity is associated with the anticorrelated behavior. This relationship between the correlation and fitness is better elucidated by plotting their relationship, as shown in Fig. 2(b), where the color is graded from dark to light for high to low confidence in the correlation result. We see a steady change in the correlation from positive to negative values that coincides with an increasing fitness value.

The discussion above shows that as the learning algorithm finds pulses that preferentially enhance the 27th harmonic, the intensity fluctuations of the 25th and 29th harmonic orders due to perturbations of the trial pulse shapes become anticorrelated. The algorithm was very effective at increasing the intensity of the 27th harmonic by nearly an order of magnitude, by means of a process we have termed intraatomic phase matching (IAPM), described elsewhere [14]. As explained above, the HHG emission phase is determined by the quantum phase that the electron wave packet accumulates during its free trajectory in the laser field, and depends on the integral history of the amplitude and phase over the fraction of the driving laser pulse when the electron is free. Thus, by controlling the laser pulse shape with sub-opticalcycle sensitivity, we control the recollision times of the electron wave function during different half cycles of the laser pulse. This controls the phase of the harmonic emission from each half cycle. A properly sculpted driving pulse will ensure that the 27th-harmonic radiation generated in each half-cycle of the driving field interferes constructively for the $\sim$ ten half

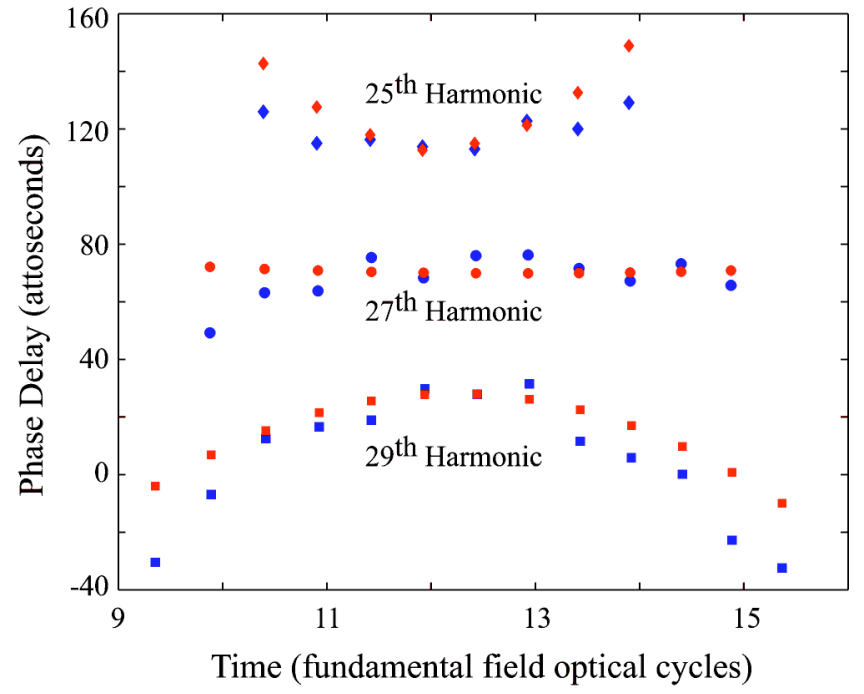

FIG. 3. Physical mechanism behind the anticorrelated intensity behavior observed for neighboring harmonic orders with small pulse shape perturbations about the optimal pulse shape for selective optimization of the 27 th order. Black points: calculated distribution of the harmonic emission phases for each recollision event for an optimal pulse shape that can select a single harmonic order. In this case, the target harmonic order phases are well aligned (maximum harmonic intensity) while neighboring orders have opposite signs of curvature (yielding lower intensity harmonics). Gray points: a slight perturbation in the laser pulse shape has little effect on the target harmonic. However, the higher (29th) harmonic order emission from each recollision event becomes more in phase, which will lead to an increase in intensity, while the lower harmonic order (25th) will decrease in intensity. This will result in an anticorrelation in the brightness of neighboring harmonic intensities in response to pulse shape perturbations around the optimal pulse shape.

cycles that contribute to the harmonic. This increases the intensity of the 27th order. On the other hand, simulations also predict that for the same optimized pulse shape, the adjacent 25th and 29th harmonic orders are generated with a phase variation of hundreds of attoseconds and with opposite chirp. These calculations are shown in Fig. 3 (black points).

The ability to enhance HHG using shaped light pulses via IAPM is consistent with our understanding of the physics of HHG. During the leading edge of a transform-limited laser pulse, the increasing laser intensity leads to the generation of harmonics with increasing values of negative chirp, as a result of the intensity-dependent phase acquired by the propagating electron $[15,16]$. This chirp can be partially compensated for by adjusting the chirp of the laser. However, only by finding a nonlinearly chirped laser pulse using the learning algorithm can a relatively flat harmonic phase and significant enhancements of the harmonic intensity be obtained $[2,13,14]$. Moreover, because the harmonic phase increases slightly from harmonic to harmonic, the optimal laser pulse can achieve selective optimization of a single harmonic order. Finally, since the harmonic chirp varies monotonically with increasing harmonic order, if one harmonic is optimized for flat phase, then adjacent harmonics will have opposite chirps. As a result, small variations in laser pulse chirp (as a result of small variations in laser phase about the optimal 
pulse shape to select a single harmonic order) can lead to an anticorrelated intensity behavior for the adjacent harmonic orders. Thus the data of Figs. 1 and 2 further validate the IAPM mechanism.

The physical mechanism behind the anticorrelated behavior for the adjacent harmonic orders is show in Fig. 3, which shows the harmonic phases corresponding to the optimal pulse (black points) as well as those corresponding to an example perturbation (gray points) about the optimal point. As the learning algorithm probes parameter space to reshape the driving laser pulse after the optimized pulse shape is found, it effectively shifts the relative timing of the electron recollision events by small amounts. This timing shift is similar for all harmonic orders. Pulse shape perturbations that make a particular recollision event become more out of phase for the 25th order will move the timing of that recollision event more in phase for the 29th order, and vice versa as indicated in Fig. 3 (gray points). Therefore, the intensities of the 25th and 29th orders should be anticorrellated, for some small pulse shape perturbations about the optimal point for selective enhancement of the 27th order. Thus a statistical analysis of the data can validate the mechanism for optimization. This behavior was not known or predicted previously; but rather was discovered by the optimization process, both experimentally and theoretically, and validated by the statistical behavior of the data. Previously, there was no mechanism for selective optimization of a single harmonic order, or for imparting different phases and chirps on adjacent harmonic orders, or for observing anticorrelated intensity behavior.

We also carried out numerical simulations of the learning experiment and performed identical statistics on the simulated data, in order to show that both experiment and theory reproduce the same statistical behavior and to further validate the IAPM mechanism. Our numerical models for high harmonic generation are described elsewhere [14]. Figure 4(a) shows the calculated peak intensity of the 25th, 27th, and 29th harmonic orders for each trial pulse shape from each iteration of the learning algorithm simulation. The correlation between the 25th and 29th harmonic orders from the entire set of simulated data is plotted in Figs. 4(b)-4(d) for the first, second, and third fractions of the data set. These data demonstrate that the statistical changes in the 25th and 29th harmonic intensities are similar to that observed experimentally. Note that because of the different number of theory data points, different initial conditions, noise levels and mutation rates in the experiment and theory, the appearance of Figs. 1 and 4 are somewhat different. This is also expected because the theoretical phases that are added to the pulse can be more varied than the experimental values, because of the imperfect nature of the pulseshaper that likely limits the available pulse shapes to those with relatively low-order phase variations. This will lead to a more pronounced anti correlation behavior for the experimental data, since many high-order phase variations will not lead to flatter phase for one harmonic and thus will not exhibit any anticorrelation in the intensities.

Figure 5(a) shows the moving window Spearmann's rho for the simulated data; note the window width of 100 was chosen to maximize the average confidence value across the

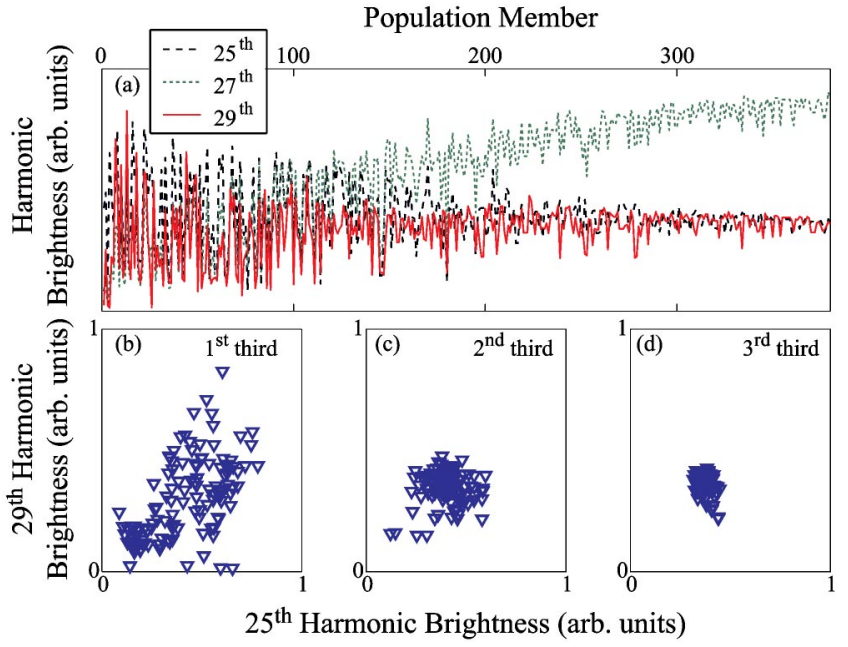

FIG. 4. (a) Peak brightness of the 25th, 27th, and 29th, harmonic orders from a learning algorithm simulation designed to selectively optimize the 27th harmonic. A correlation map of the 25th and 29th harmonic orders for the first (b), second (c), and third (d) fractions of the learning algorithm simulation.

data set. The anticorrelated behavior [Fig. 5(a), triangles], is evident in the simulation as the optimization proceeds, and is again accompanied by an increase in the fitness [Fig. 5(a), points], indicating selectivity of harmonic orders occurs for pulse shapes that exhibit anticorrelated behavior. The relationship between the correlation and fitness for the simulated data is shown in Fig. 5(b), where again the color is graded from dark to light for high to low confidence in the correlation result. The agreement between the statistical behavior of experiment and theory in Figs. 2 and 5 demonstrates the use of learning algorithms to uncover interesting and unexpected dynamics in complex quantum systems.

When comparing experiment and theory from Figs. 2(b)

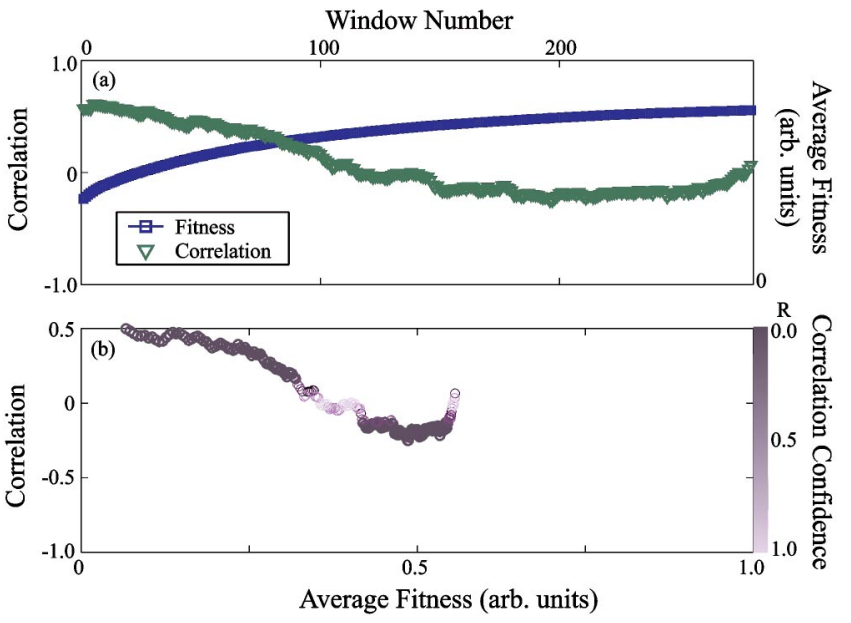

FIG. 5. (a) Correlation of the 25th and 29th theoretical harmonic brightness as a moving window is scanned along algorithm generations (triangles) and the corresponding average fitness value (points). (b) Correlations plotted vs the average fitness value for the data in (a), where the color is graded from dark to light for high to low confidence in the correlation result. 
and 5(b), the dark data points should be used. These are the data points for which there is a high degree of confidence in the correlation result. Experimentally in Fig. 2(b), the 25th and 29th harmonic orders start out slightly correlated, and become strongly anticorrelated. Theoretically in Fig. 5(b), the 25th and 29th harmonic orders start out correlated, and become anticorrelated. The difference between theory and experiment at the start of the optimization can be explained by varying initial conditions, and small positive correlations could be due to overall pulse duration and intensity fluctuations. At the end of the optimization (which can occur over a different number of iterations for theory and experiment), when the fitness is high indicating selective optimization, the 25th and 29th harmonics show anticorrelated behavior for both theory and experiment for those points (dark) that have a high degree of confidence in the correlation results. Thus there is good agreement between theory and experiment, even in the detailed statistical behavior of the optimization. One would not expect perfect agreement because the noise levels, mutation rates, and accessible pulse shapes are not the same for theory and experiment.

In conclusion, this analysis represents a different demonstration of extracting the temporal dynamics of a high-field quantum process by studying the ensemble behavior of the populations involved in a learning control optimization procedure. Analyses of the complete data sets were used to study the global behavior of the attosecond dynamics and control of free electron wave packets and to validate the mechanism behind selective optimization of high-harmonic generation. Moreover, the learning control algorithm automates the study of the parameter space, and selects an interesting and useful region for investigation.

The authors gratefully acknowledge support for this work from the National Science Foundation and from the Chemical Sciences, Geosciences and Biosciences Division of the Office of Basic Energy Sciences, U.S. Department of Energy.
[1] P. Barbara, J. Fujimoto, W. Knox, and W. Zinth, Ultrafast Phenomena X (Springer-Verlag, Berlin, 1996).

[2] R. Bartels, S. Backus, E. Zeek, L. Misoguti, G. Vdovin, I. P. Christov, M. M. Murnane, and H. C. Kapteyn, Nature (London) 406, 164 (2000).

[3] G. G. Paulus, F. Grasbon, H. Walther, P. Villoresi, M. Nisoli, S. Stagira, E. Priori, and S. De Silvestri, Nature (London) 414, 182 (2001).

[4] M. Drescher, M. Hentschel, R. Kienberger, G. Tempea, C. Spielmann, G. A. Reider, P. B. Corkum, and F. Krausz, Science 291, 1923 (2001).

[5] P. M. Paul, E. S. Toma, P. Breger, G. Mullot, F. Auge, P. Balcou, H. G. Muller, and P. Agostini, Science 292, 1689 (2001).

[6] H. Rabitz, R. de Vivie-Riedle, M. Motzkus, and K. Kompa, Science 288, 824 (2000).

[7] D. Meshulach and Y. Silberberg, Nature (London) 396, 239 (1998)

[8] D. Oron, N. Dudovich, D. Yelin, and Y. Silberberg, Phys. Rev. Lett. 88, 063004 (2002).

[9] R. J. Levis, G. M. Menkir, and H. Rabitz, Science 292, 709 (2001).

[10] J. L. Herek, W. Wohlleben, R. J. Cogdell, D. Zeidler, and M. Motzkus, Nature (London) 417, 533 (2002).

[11] A. Assion, T. Baumert, M. Bergt, T. Brixner, B. Kiefer, V. Seyfried, M. Strehle, and G. Gerber, Science 282, 919 (1998).

[12] T. C. Weinacht, R. Bartels, S. Backus, P. H. Bucksbaum, B. Pearson, J. M. Geremia, H. Rabitz, H. C. Kapteyn, and M. M.
Murnane, Chem. Phys. Lett. 344, 333 (2001).

[13] R. Bartels, S. Backus, I. Christov, H. Kapteyn, and M. Murnane, Chem. Phys. 267, 277 (2001).

[14] I. P. Christov, R. Bartels, H. C. Kapteyn, and M. M. Murnane, Phys. Rev. Lett. 86, 5458 (2001).

[15] Z. Chang, A. Rundquist, H. Wang, I. Christov, H. C. Kapteyn, and M. M. Murnane, Phys. Rev. A 58, R30 (1998).

[16] P. Salieres, P. Antoine, A. de Bohan, and M. Lewenstein, Phys. Rev. Lett. 81, 5544 (1998).

[17] E. Gibson, A. Paul, N. Wagner, S. Backus, M. M. Murnane, H. C. Kapteyn, R. A. Bartels, and I. P. Christov, in 3rd International Workshop on Optimal Control of Quantum Dynamics: Theory and Experiment (Ringberg, Germany, 2003).

[18] J. L. White, B. J. Pearson, and P. H. Bucksbaum, e-print quantph/0401018.

[19] C. Daniel, J. Full, L. Gonzalez, C. Lupulescu, J. Manz, A. Merli, S. Vajda, and L. Woste, Science 299, 536 (2003).

[20] E. Zeek, R. Bartels, M. M. Murnane, H. C. Kapteyn, S. Backus, and G. Vdovin, Opt. Lett. 25, 587 (2000).

[21] A. Rundquist, C. G. Durfee III, S. Backus, C. Herne, Z. Chang, M. M. Murnane, and H. C. Kapteyn, Science 280, 1412 (1998).

[22] E. Zeek, K. Maginnis, S. Backus, U. Russek, M. Murnane, G. Mourou, H. Kapteyn, and G. Vdovin, Opt. Lett. 24, 493 (1999).

[23] M. Hollander and D. A. Wolfe, Nonparametric Statistical Methods (Wiley, New York, 1973). 